\title{
Isolated Pancreatic Metastasis From Melanoma: A Case Report
}

Solmaz A', Yiğitbaş $H^{1}$, Tokoçin $M^{1}$, Gülçiçek OB', Erçetin $C^{1}$, Yavuz $E^{1}$, Altınay $S^{2}$, Tetikurt $S^{2}$, Çelebi $F^{1}$ and Çelik $A^{1}$

'Department of General Surgery, Bagcilar Training and Research Hospital, Istanbul, Turkey

${ }^{2}$ Department of Pathology, Bagcilar Training and Research Hospital, Istanbul, Turkey

Corresponding author: Serdar Altınay, M.D. Bağcılar Eğitim ve Araştırma Hastanesi, Patoloji Laboratuvarı, Merkez Mahallesi Mimar Sinan Caddesi No:6. Bağcılar, Istanbul, Türkiye 34203 , Tel: +90 (0) 212-440 4000 / 3328; Fax: +90 (0) 21244042 43; E-mail: drserdara@yahoo.com

Received date: Sep 24, 20134, Accepted date: Nov 27, 2014, Published date: Nov 30, 2014

Copyright: (c) 2014 Solmaz A, et al. This is an open-access article distributed under the terms of the Creative Commons Attribution License, which permits unrestricted use, distribution, and reproduction in any medium, provided the original author and source are credited.

\begin{abstract}
Malignant cutaneous melanomas are skin tumours with a worse prognosis. Presence of metastasis is an indicator of a very aggressive nature of melanoma. Though it can be rarely seen as a primary tumour of the gastrointestinal system, it is among the most frequently observed metastatic tumor of the gastrointestinal system. Since isolated pancreatic metastasis is rarely seen, limited information is available in the literature. In some publications, resection of isolated pancreatic metastasis has been suggestively associated with prolonged life expectancy of the patients. In our manuscript, we will present a very rarely seen case of malignant skin melanoma with isolated pancreatic metastasis.
\end{abstract}

Keywords: Melanoma; Isolated pancreatic metastasis

\section{Introduction}

Majority of malignant pancreatic tumours constitutes adenocarcinomas arising primarily from pancreatic tissue. In systemic metastases of other organ cancers, pancreas is frequently affected, while isolated pancreatic metastasis comprises $2 \%$ of all pancreatic cancers [1]. Mostly kidney, breast, lung and colonic cancers and more rarely melanomas and sarcomas metastasize to pancreas. Nearly $6 \%$ of all isolated pancreatic cancers metastasize from malignant melanomas [2].

Malignant melanomas are skin tumors with an aggressive course. Melanomas frequently metastasize to gastrointestinal system (in autopsy series $50-60 \%$ of the cases) [3]. Metastasis to pancreas from any organ is generally considered as a worse prognostic factor and the patient is thought to be in his/her terminal phase of the disease. However, in recent years, in cases with isolated pancreatic metastases, metastasectomy has been applied and this operation is known to increase life span and quality of life [4].

Longevity of life expectancy is approximately 6-12 months in cases with melanomas metastasized into visceral organs [5]. Response rates to chemotherapy changes between 15 and $28 \%$ with a long-term remission rate of about 2 percent [6].

\section{Case}

A 59-year-old male patient consulted to our clinic with complaint of abdominal pain. Laboratory tests revealed higher blood liver enzymes and bilirubin values. On upper abdominal ultra-sonograph, sludge in the gallbladder, dilation of intrahepatic, extrahepatic and common biliary ducts $(14 \mathrm{~mm})$ were detected. On abdominal computed tomography obtained, dilated intra- and extrahepatic biliary ducts, abrupt and blunt common bile duct termination and a mass lesion nearly $38 \mathrm{~cm}$ in diameter were observed in the head of the pancreas (Figure 1).

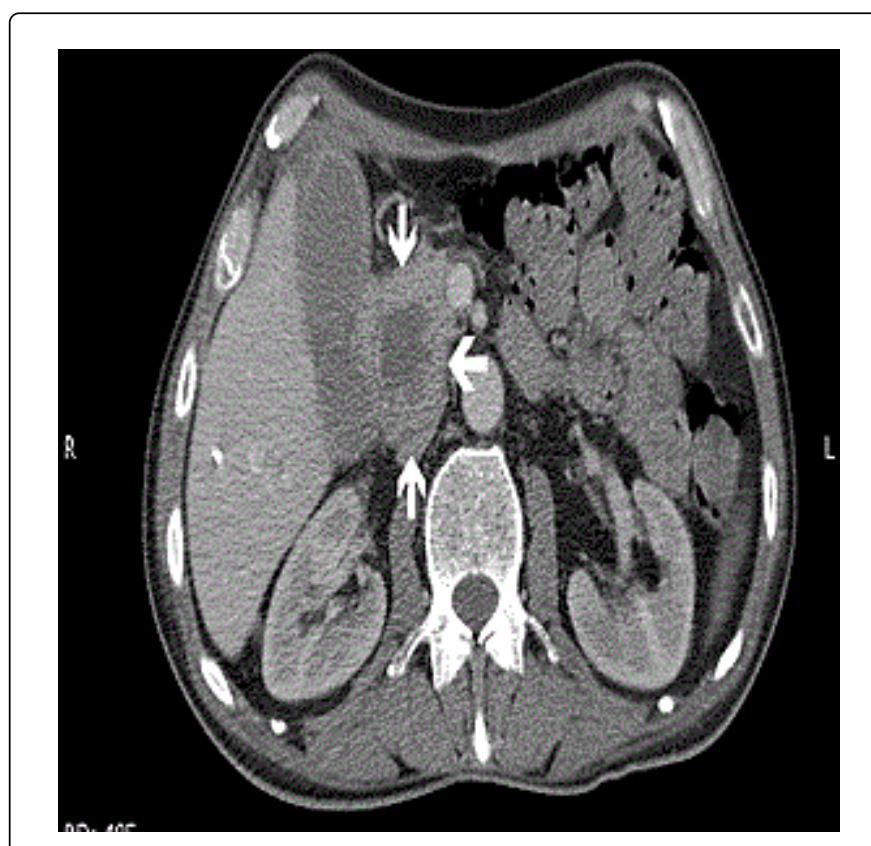

Figure 1: A computerized axial tomography of the abdomen demonstrating tumoral mass (white arrows) in the head of the pancreas.

We learnt from his medical history that nearly 1.5 years ago he had a nevus on his right forearm which was excised. Then its histopathology was reported as Clark's level 5 (Invasion into the subcutaneous fat) melanoma. He had received interferon therapy for nearly one year after excision of the melanoma. In the light of this information, pancreatic biopsy was planned for the patient. In histopathological examination of pancreatic biopsy specimen, metastasis from melanoma was detected (Figure 2). Following radiological evaluation, the mass lesion was considered to be 
irresectable, and then percutaneous trans-hepatic cholangiography was planned. However, the patient did not accept interventional procedures so the procedure was cancelled. The patient was directed to the department of medical oncology and lost to follow-up.

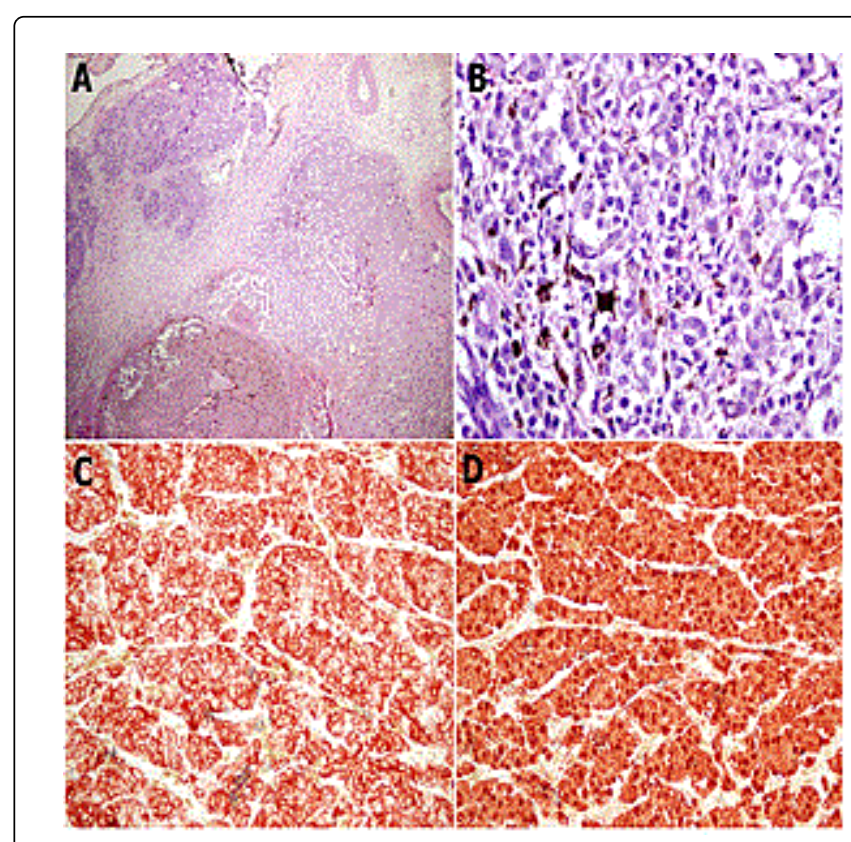

Figure 2: Microscopic appearance of the pancreatic biopsy specimen. (A); Scarce amount of pancreatic tissue adjacent to atypical cell proliferation in a solid nodular pattern (H\&E;x40). (B); Pleomorphic atypical tumour cells with occasional bizarre nuclei and eosinophilic cytoplasm are seen. Distinct melanin pigment in the center is remarkable. (H\&E; $x 400)$. (C); Strong HMB-45 immunoreactivity of the tumour cells is observed (H\&E;x200). (D); Strong S-100 immunoreactivity of the tumour cells is seen (H\&E;x200).

\section{Discussion}

Malignant melanoma is the most fatal one among all skin cancers and related mortality rates are increasing rapidly. Remote metastases are most frequently seen in the lungs, liver, brain and gastrointestinal system. In primary skin melanomas, the most important factors effecting patient's survival are depth of the tumour, ulceration, histological subgroup, its location and lymph node involvement. Based on these parametres, Clark and Breslow classifications have been used for melanomas. The Clark level is based on a one through five level depths of invasion and based on the separate levels of the skin. Primary cutaneous melanoma thickness, ulceration, and stage were identified and validated as risk factors for the metastasis [7].

Primary melanoma of the gastrointestinal tract is very rarely seen. Skin melanomas mostly metastasize to the stomach in the gastrointestinal system. In an autopsy series of 216 patients, among gastrointestinal system organs, the incidence rates of metastasis to liver (58.3\%), peritoneum (42.6\%), pancreas (37.5\%), small intestines (35.6\%), spleen $(30.6 \%)$, colon $(28.2 \%)$ and stomach $(22.7 \%)$ were reported as indicated [8].
Gastrointestinal metastases of melanomas generally do not give clinical symptoms or become symptomatic at a late stage. However, metastases can be detected during radiological examinations performed for routine control of the patients followed up for their primary disease. In the presence of metastases, radiological examinations, endoscopic procedures which enable biopsy or Positron Emission Tomography (PET) are mainly applied diagnostic methods [9]. Since PET can fail to demonstrate metastatic lesions of melanoma less than $1 \mathrm{~cm}$ in diameter, it should be used in combination with other radiological modalities.

Medical past of the patient with a mass lesion in the head of the pancreas should be inquired as for malignancies of other organ systems. Ultrasound is the first diagnostic imaging modality to be resorted to in patients with a suspect pancreatic mass who had been previously treated for other organ cancers. Based on ultrasonographic findings, contrasted abdominal CT gives an idea about contrast uptake of the mass lesion, its relationship with adjacent organs and its respectability [10]. CT scan is not always helpful in differentiating primary from secondary pancreatic lesions. Pancreatic metastasis may present as solid or cystic, hypodense or hyper dense lesions. CT findings of pancreatic tumours are described suggested that multiplicity of tumours and/or hypervascularity were characteristic of secondary pancreatic tumours [11].

Median life expectancy of the patients after development of gastrointestinal metastases ranges between 6 and 12 months. In Clark $\geq 4$ level (tumoral infiltration into dermis) melanomas, surgical intervention is used only for palliation. Rarely, surgical excision of isolated distant metastases has provided survival advantage. In a study Wood et al. [12] demonstrated 5-year survival rates for patients who had or had not undergone pancreatic resection as $37.5 \%$ (median survival time, 23.8 months) and 23\% (median survival time, 15.2 months), respectively [12]. However limited experience is available for still debatable pancreatic resection applied for metastatic melanoma. The benefits of surgery for patient to increase survival rate has been observed for metastases from Renal cell carcinoma, while for other primary tumors the role of surgery is mainly palliative [13].

Primary treatment modality in malignant melanomas is surgery. Chemotherapy and immunotherapy have a limited place in the treatment protocol. In a study by Lee et al. in patients with pancreas metastasis from melanoma, pre- and post-operative chemotherapy did not change life expectancy of the patients significantly [14].

\section{Conclusion}

In patients with pancreatic mass lesions, tumours of other organs should be investigated. Metastatic lesions of melanoma should be kept in mind as a possibility especially in patients with a past history of kidney, breast or colonic cancers or melanoma and approach to these patients should be determined accordingly. Available experience is very limited on clinical and surgical experience and patient's perception of life expectancy achieved by the applied treatment modality in cases with isolated pancreatic metastasis from a melanoma. Many studies performed have suggested that total surgical excision of the lesion prolongs life expectancy.

\section{Conflict of Interest}

- The authors of the article certify that they have NO affiliations with or involvement in any organization or entity with any financial 
Citation: Solmaz A, Yigitbas H, Tokocin M, Gulcicek OB, Ercetin C, et al. (2014) Isolated Pancreatic Metastasis From Melanoma: A Case Report. J Carcinog Mutagen 5: 202. doi:10.4172/2157-2518.1000202

Page 3 of 3

interest or non-financial interest in the subject matter or materials discussed in this manuscript.

- Written informed consent was obtained from all patients, the parents or legal guardians.

\section{References:}

1. Goyal J, Lipson EJ, Rezaee N, Edil BH, Schulick R, et al. (2012) Surgical resection of malignant melanoma metastatic to the pancreas: case series and review of literature. J Gastrointest Cancer 43: 431-436.

2. He MX, Song B, Jiang H, Hu XG, Zhang YJ, et al. (2010) Complete resection of isolated pancreatic metastatic melanoma: a case report and review of the literature. World J Gastroenterol 16: 4621-4624.

3. Schuchter LM, Green R, Fraker D (2000) Primary and metastatic diseases in malignant melanoma of the gastrointestinal tract. Curr Opin Oncol 12: 181-185.

4. Chua TC, Saxena A (2010) Pancreatic cancer margin status after pancreaticoduodenectomy-the way forward. Ann Surg 251: 775-776.

5. Balch CM, Soong SJ, Gershenwald JE, Thompson JF, Reintgen DS, et al. (2001) Prognostic factors analysis of 17,600 melanoma patients: validation of the American Joint Committee on Cancer melanoma staging system. J Clin Oncol 19: 3622-3634.

6. Reddy S, Wolfgang CL (2009) The role of surgery in the management of isolated metastases to the pancreas. Lancet Oncol 10: 287-293.
7. Nikfarjam M, Evans P, Christophi C (2003) Pancreatic resection for metastatic melanoma. HPB (Oxford) 5: 174-179.

8. Patel JK, Didolkar MS, Pickren JW, Moore RH (1978) Metastatic pattern of malignant melanoma. A study of 216 autopsy cases. Am J Surg 135: 807-810.

9. Liang KV, Sanderson SO, Nowakowski GS, Arora AS (2006) Metastatic malignant melanoma of the gastrointestinal tract. Mayo Clin Proc 81: 511-516.

10. Portale TR, Di Benedetto V, Mosca F, Trovato MA, Scuderi MG, et al. (2011) Isolated pancreatic metastasis from melanoma. Case report. G Chir 32: 135-137.

11. Klein KA, Stephens DH, Welch TJ (1998) CT characteristics of metastatic disease of the pancreas. Radiographics 18: 369-378.

12. Eidt S, Jergas M, Schmidt R, Siedek M (2007) Metastasis to the pancreas-an indication for pancreatic resection? Langenbecks Arch Surg 392: 539-542.

13. Sperti C, Moletta L, Patanè G (2014) Metastatic tumors to the pancreas: The role of surgery. World J Gastrointest Oncol 6: 381-392.

14. Lee CC, Faries MB, Wanek LA, Morton DL (2009) Improved survival for stage IV melanoma from an unknown primary site. J Clin Oncol 27: 3489-3495. 